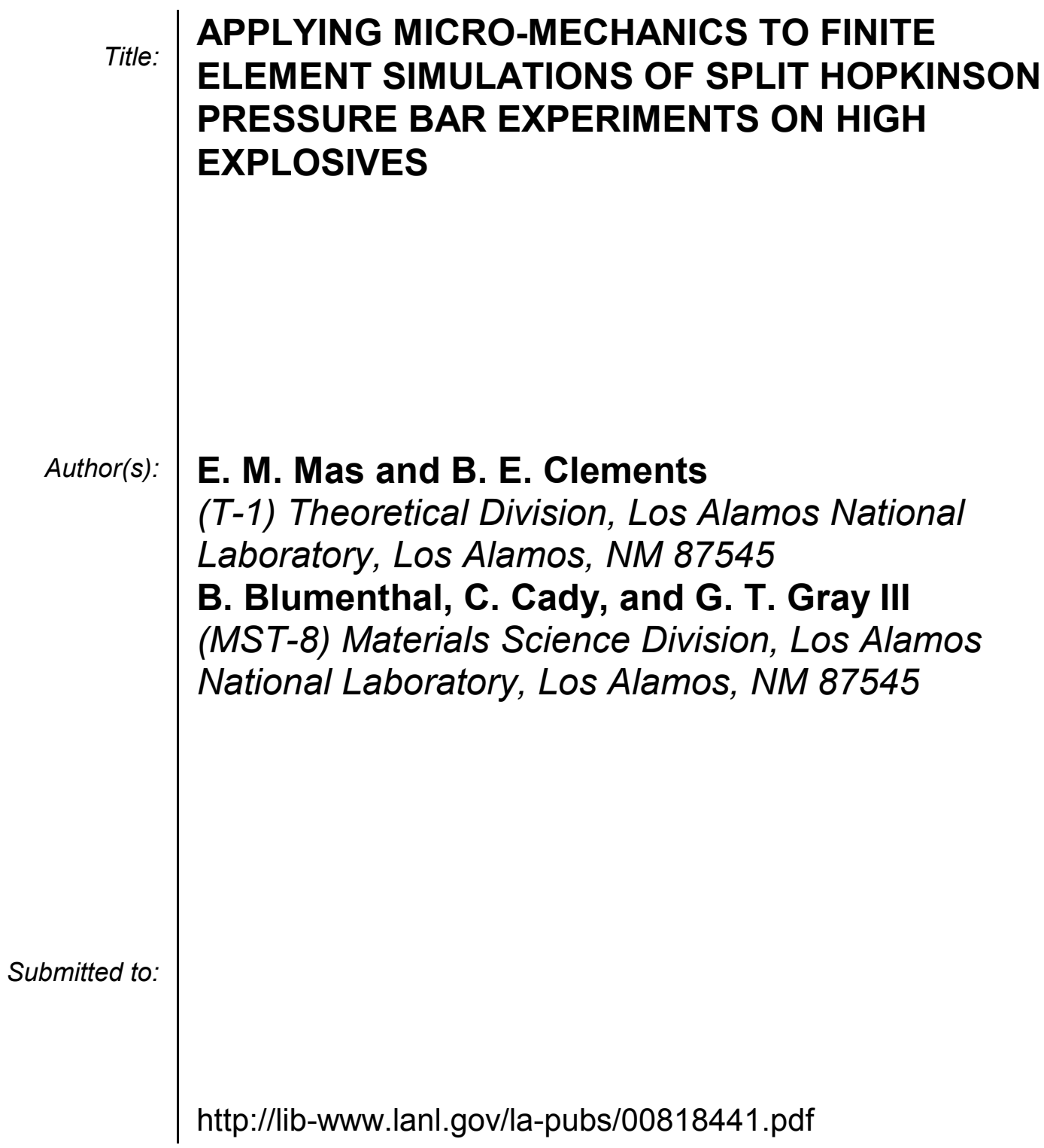




\title{
APPLYING MICRO-MECHANICS TO FINITE ELEMENT SIMULATIONS OF SPLIT HOPKINSON PRESSURE BAR EXPERIMENTS ON HIGH EXPLOSIVES
}

\author{
E. M. Mas and B. E. Clements \\ (T-1) Theoretical Division, Los Alamos National Laboratory, Los Alamos, NM 87545
}

B. Blumenthal, C. Cady, and G. T. Gray III

(MST-8) Materials Science Division, Los Alamos National Laboratory, Los Alamos, NM 87545

\begin{abstract}
We have developed a constitutive theory based on the Method of Cells and a modified Mori-Tanaka (MT) effective medium theory to model high explosives. MT effective medium theory allows us to model the smaller explosive grains in the viscoelastic matrix while the Method of Cells partitions the representative volume element into a single subcell designating a large grain, and the remaining subcells for the small grain-binder mixture. The model is then implemented into the finite-element code EPIC. Split Hopkinson Pressure Bar (SHPB) experiments are simulated. We compare the predicted incident, transmitted and reflected strains with SHPB experimental values. [Research supported by the USDOE under contract W-7405-ENG-36.]
\end{abstract}

\section{INTRODUCTION}

Modeling composites with micro-structures involving a wide range of length scales is a continuing problem in mechanics of materials studies. We have proposed a constitutive theory for filled-polymer composites. This homogenization technique allows us to model an isotropic rubbery polymer matrix filled with uniformly distributed elastic filler particles (or voids) of arbitrary aspect ratio, either aligned or randomly orientated. In this work we couple this theory with the Method of Cells (MOC) in an attempt to model the complex micro-structure of PBX-9501.

We will show that by incorporating viscoelasticity, plasticity, and cracking into our model we will be able to reproduce the mechanical properties of PBX-9501 at strain rates ranging from quasi-static to plate impact type rates $\left(10^{6} \mathrm{~s}^{-1}\right)$ with particular emphasis on the rates of $10^{3} \mathrm{~s}^{-1}$ achieved with Split Hopkinson Pressure Bar (SHPB) experiments. These experiments have been simulated in 2-D using the finite element (FEM) code EPIC ${ }^{1}$.

\section{THE MODEL OF PBX-9501}

The microstructure of PBX 9501 is complex, with HMX grain sizes ranging from hundreds of microns to less than one micron. The MOC technique was chosen because when used with our composite theory it is able to accommodate these desperate length scales. Since Ref. 2 provides an excellent description of the MOC, we provide only an outline here.

The theory hinges on the ability to create a representative volume element (RVE) that describes to overall structure of the composite. We use a simple $(2 \times 2 \times 2)$ RVE with the composite theory since our goal is to use these methods in large scale FEM simulations. The large crystals are modeled by filling a large subcell in the RVE with pure HMX. The large cell is chosen to represent approximately 90 volume percent of the RVE. The remaining 
seven cells are filled with a homogenized mixture of HMX and plasticized estane binder. The mixture consists of 40 volume percent HMX, which brings the total volume percent of HMX to the desired value of $93.5 \%$. We applied our filled polymer theory described in Ref. 3 to model the filled matrix. Since the theory is thoroughly presented in those references we will only provide a cursory description.

Our theory is based on an expansion about the ratio of shear modulus to bulk modulus, which is presumed to be small. We define this ratio as $y$ and refer to the Taylor expansion about $y=0$ as the $y$ expansion. While the polymer is in the rubbery phase, its shear modulus is often orders of magnitude smaller than its bulk modulus. Only at extremely high loading rates or temperatures below the glass transition temperature will $y$ approach or exceed one. Therefore, as long as we are in the rubbery phase of the polymer our assumption of small $y$ will be valid. Other assumptions are that the bulk modulus is much less rate dependent than the shear modulus and the polymer is isotropic. As a consequence of the former we assume that the bulk modulus is rate and temperature independent. This restriction can be removed if more information were available.

Applying our y-expansion to Mori and Tanaka's ${ }^{4}$ effective medium theory and expressions for the composite's effective elastic stiffness tensor derived by Weng and coworkers ${ }^{5}$ we derive expressions which can be analytically inverse Laplace transformed to produce closed expressions for the composites stress relaxation moduli with coefficients which are functions of the filler concentration, ellipsoidal aspect ratio, and moduli. If the $y$ expansion were not carried out the inverse Laplace transforms would have to be conducted numerically. Since this is notoriously unstable, it is highly desirable to circumvent this step, especially since the goal is a constitutive theory applicable to large-scale finite element method simulations where many such transformations must be done.

\section{CONSTITUTIVE LAWS}

The HMX crystals are treated as elastic in this model, while the plasticized estane binder is viscoelastic. Further, we allow for the large HMX crystals to crack, which has been observed experimentally. ${ }^{6}$ Plastic yield has been applied at the von Mises level for all subcells. Viscoelasticity of the HMX could also be implemented, but since there is little data on which to base a model, it has been ignored. Further, debonding between the large HMX crystal and the composite binder can also be included, but since the SHPB experiment is exclusively compressive, it would have little effect, and we only mention it here to highlight the versatility of the model.

The elastic moduli, shear and bulk, were taken to be 5.4 and $12.5 \mathrm{GPa}$ respectively. These values are used for both the small filler particulates used in the continuum model with the plasticized estane and for the large crystal in the large subcell of the RVE in the MOC.

Using the master curves of the storage shear modulus as a function of rate times the $\mathrm{WLF}^{7}$ shift, and the shift as a function of temperature created by G. L. Flowers ${ }^{8}$ we have created a 22 element Maxwell model to represent the viscoelastic response of the plasticized estane. We note here that we have used the time-temperature superposition principle, which is the source of the above mentioned shifts, but this is not necessary when using our MOC/filled polymer technique and the use of tables or any other way of modeling the viscoelastic response could be applied. Ref 9 provides further details.

We used the crack growth model of Johnson, Addessio, and Dienes. ${ }^{10}$ The model is represented by the equation:

$$
2 G \dot{\varepsilon}_{i j}^{c}=\left(\frac{c}{a_{0}}\right)^{3} s_{i j}
$$

where $G$ is the shear modulus, $\dot{\varepsilon}_{i j}^{c}$ is the cracking contribution to the $i j^{\text {th }}$ component of the strain, $c$ is the crack diameter, $a_{0}$ is an empirical size factor, and $s_{i j}$ is the stress deviator. There are two (smoothly connected) crack growth regimes, slow and fast. The onset of fast crack growth is defined by the empirical parameter $K_{0}$. The $\eta_{\max }$ parameter is some value less than the shear wave speed and is the value to which the crack growth rate asymptotically approaches. The free parameters $K_{0}$, $\eta_{\max }, a_{0}$, and $v^{\exp }$ (which describes the shape of the slow crack growth curve), were chosen by a rough optimization with respect to quasi-static, SHPB, and plate impact experiments to be 438 PSI (in) ${ }^{1 / 2}$, $0.302 \mathrm{~cm} / \mu \mathrm{s}, 0.692 \mathrm{~mm}$, and 5.266 respectively.

As a first attempt we have applied a uniform von Mises model to all subcells of the RVE. We note that we could have applied yielding to the constituents directly, but due to the lack of available 
data we choose to a value of $50 \mathrm{MPa}$ for the yield stress taken from Ref. 11 to each subcell.

\section{SIMULATIONS AND EXPERIMENTS}

Before applying our theory to more complicated multi-dimensional experiments considerable effort was devoted to comparing our theoretical predictions to experimental measurements which are effectively one dimensional. These results are presented in these proceedings, Ref. 12.

SHPB experiments were simulated using the finite element code EPIC. ${ }^{1}$ The goal of the experiment is to attain a uniaxial stress state in the sample. Due to the symmetry of the apparatus all of our EPIC simulations were conducted in two dimensions (cylindrical symmetry). An illustrations of the grid used is provided in Fig. 1. We found that a striker speed of 515 inches per second (ips) created a strain wave in the Magnesium incident bar with an amplitude of $0.14 \%$, a good approximation of the experimental value (see the first pulse in Fig. 2). This adjustment was necessary because the striker speed is not measured in the experiment. As can be seen in Fig. 2 our simulation produces very acceptable raw strain curves at the gauge positions.

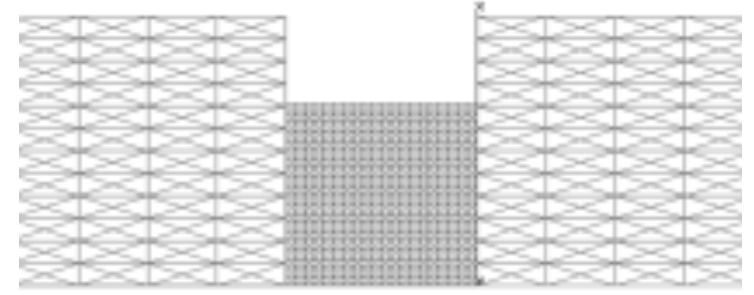

FIGURE 1. The grid used in the finite element simulation

Figure 3. compares the stress-strain curves reduced from the experimental raw curves using the 1-wave analysis ${ }^{13}$ to our simulated values. We have also included a simulation without cracking. It is apparent that cracking is responsible for the "chewing" away of the wave, creating the observed negative slope in the stress-strain curve. Apart from an apparent mismatch at the origin, the simulation with cracking follows the two experimental curves very well. We note that absolute times of the experiment are not measured and seating issues can shift the origin of the experimental curve.

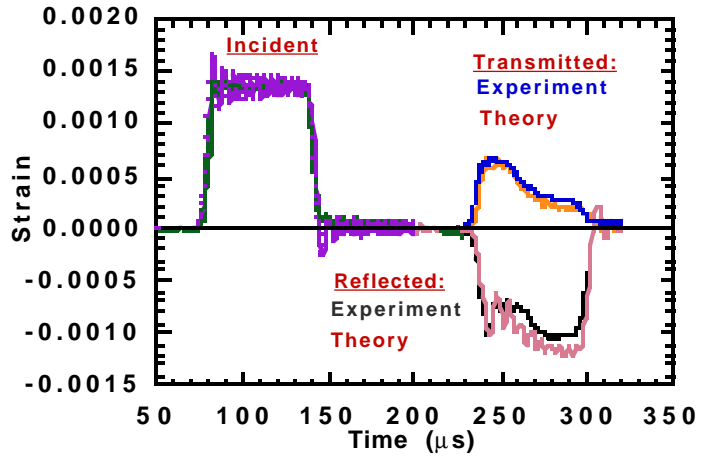

FIGURE 2. Comparison of the measured and simulated strains at the gauge positions.

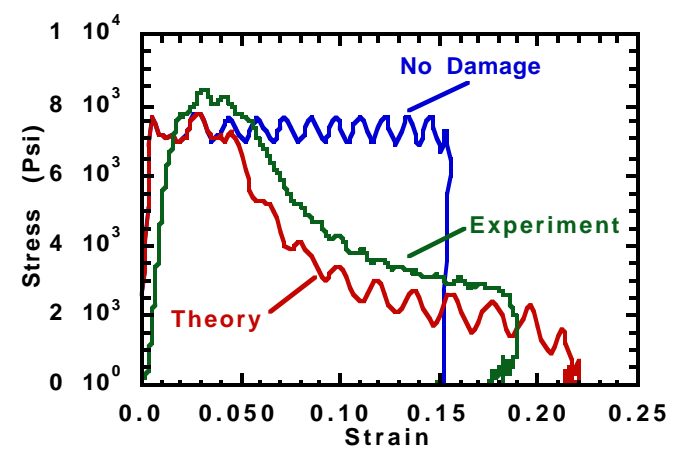

FIGURE 3. Stress-strain curves of the sample.

Figure 4 shows the strain rate as a function of stress within the sample. Again, we see a good comparison of theory with experiment. We also observe that cracking has the expected effect of softening the sample, manifested in the figure by the steady increase of the strain rate with stress, an observation reflected in the experimental curve.

A concern at this point was what effect the cracking was having on the assumptions used in reducing the raw experimental strain curves in the bars to the stress-strain and stress-strain rate curves in the sample by the 1-wave analysis. In Fig. 5 we show a plot of the volumetric strain (V/Vo-1) versus time for an element in the sample. As can be seen, when cracking is ignored the volume is constant after the initial "ring-up" (specifically due to the initial elastic response of the sample). When, however, cracking is accounted for, the volume slowly relaxes to its initial value. Therefore, strictly speaking, volume is not conserved in SHPB experiments on PBX-9501, but the change is so 
small and so gradual that it only changes the sample stresses by a couple tenths of a percent, well below the uncertainty of any simulation or experiment.

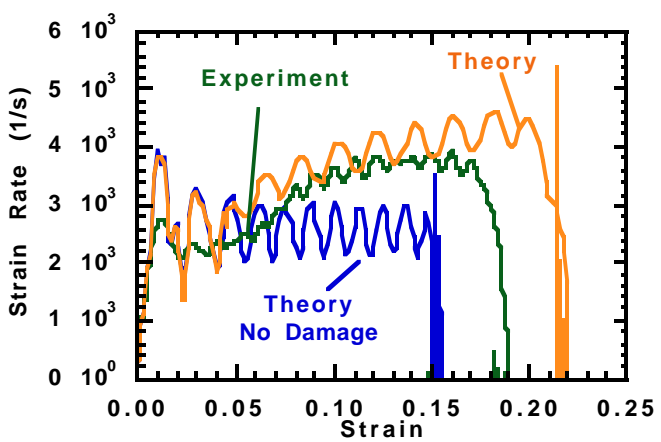

FIGURE 4. Stress versus strain-rate curves of the PBX-9501 sample reduced from experiment versus theory curves with and without cracking.

\section{CONCLUSION}

We have demonstrated that coupling the MOC with our homogenization technique sufficiently models the microstructure and hence the mechanical properties of PBX-9501 for strain rates ranging from quasi-static to $10^{6} \mathrm{~s}^{-1}$. We have also shown that cracking plays a critical role in the mechanical response in the SHPB experiment, and that while the cracking does not strictly conserve volume, it introduces minimal errors into the 1-wave data reduction of the experimental values.

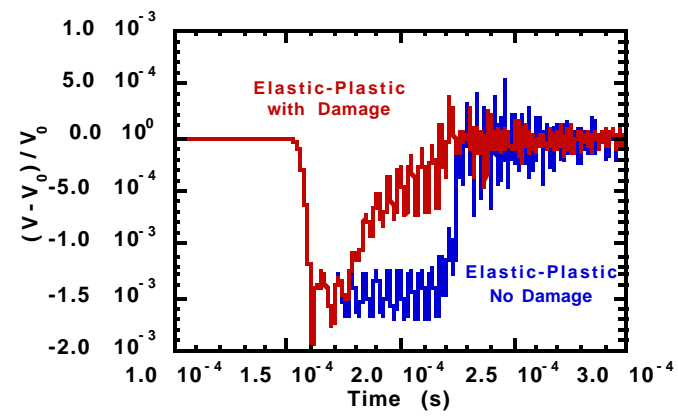

FIGURE 5. Volumetric strain of a sample element with and without accounting for cracking.

\section{ACKNOWLEDGMENTS}

The authors gratefully acknowledge financial support for this work from the Joint DoD/DOE Munitions Technology Development Program.

\section{REFERENCES}

1) Johnson, G. R., Stryk, R. A., Holmquist, T. J., and Beissel, S. R., User Instructions for the 1997 Version of the EPIC Code, Wright Laboratory, Armament Directorate, Eglin Air Force Base report, WL-TR-19977037, 1997.

2) Aboudi, J., Mechanics of Composite materials: A Unified Micromechanical Approach, Elsevier Science Publishers, Amsterdam, 1991.

3) Clements, B. E. and Mas, E. M., (private communication).

4) Mori, T. and Tanaka, K., Acta Metallurgica , 21, 571 (1973).

5) Li, J. and Weng, G. J., Mech. Mater. 22 179-188

(1995); Wang, Y. M. and Weng, G. J., J. Appl. Mech., 59, 510-518 (1992).

6) Idar, D. J., personal communication (2001).

7) Ferry, J. D., Viscoelastic Properties of Polymers 2nd Ed., Wiley, New York, 1970.

8) Flowers, G. L., Characterization of binders used in high explosives and the effect of binder strength on impact sensitivity of explosives. Report MHSMP-854, Pantex, August 1985.

9) Mas, E. M., Clements, B. E., Blumenthal, B., Cady, C., Gray, G. T. III, and Liu, C., (this Proceedings).

10) Bennett, J., Haberman, K., Johnson, J., Asay, B., and Henson, B., J. Mech. Phys. Solids, 46, 2303 (1998).

11) Dick, J. J., Martinez, A. R., and Hixson, R. S., Los Alamos National Laboratory Report, LA-13426-MS (1998).

12) Clements, B. E. and Mas, E. M., (this Proceedings). 13) Gray, G. T. III, Blumenthal, W. R., Idar, D. J., and Cady, C. M., in Shock Compression of Condensed Matter 1997, edited by S. C. Schmidt, D. P. Dandekar J. W. Forbes (American Institute of Physics, Woodbury, New York, 1997). 